\title{
Utilisation du maléate acide d'acépromazine comme tranquillisant chez les gros ruminants
}

\author{
par M. HIDIROGLOU
}

Nous avons été amené ces derniers mois à utiliser les tranquililisants sur des buffles et sur des zébus. Nous relaterons ici les observations et les commentaires qui découlent de ces essais.

\section{I. - CHEZ LES BUFFLES}

Nous avons pensé pour la première fols à recourir à l'emploi des tranquillisants afin de calmer et rendre dociles des buffles sauvages pris au piège. Nous nous sommes adressé au maléate acide d'acépromazine. Rapportons trois observa. tions typiques.

Observation $n^{\circ}$ 1. - I s'agit d'une buffilesse de $350 \mathrm{~kg}$ environ, particulièrement sauvage. Après de grosses difficultés, nous lui administrons par voie intramusculaire $200 \mathrm{mg}$ du produit à 9 heures.

A 10 heures, l'animal étant demeuré aussi méchant qu'avant l'intervention, nous lui aáministrons une nouvelle dose de $100 \mathrm{mg}$.

A II heures 30, aucun phénomène de relaxation ne s'étant produit, nous décidons d'iniecter à nouveau $100 \mathrm{mg}$.

A 11 heures 50, le tranquilisant commence enfin à agir: l'animal fonce avec une moincire vigueur sur les barrières du parc.

A 12 heures, il parait complètement apaisé : la tête et le cou baissés, les yeux mi-cios, il ne réagit plus aux excitations.

A 12 heures 30, un homme seul peut le sortir du parc et l'embarquer très facilement sur un petit bàteau.

Reçu pour publication : novembre 1959
L'effet du tranquillisant se fait sentir pendant environ 8 heures. L'animal commence à ruminer deux heures après la dernière injection.

Il nous a fallu par conséquent recourir à $400 \mathrm{mg}$ de maléate acide d'acépromazine pour relaxer un buffle de $350 \mathrm{~kg}$, soit une dose deux fois plus importante que celle indiquée par le fabricart comme dose maximum pour un bovin du même poids.

Observations $\mathrm{n}^{\mathrm{os}} \mathbf{2}$ et $\mathbf{3}$. - Elles se rapportent à deux buffles sauvages pesant respectivement environ 400 et $500 \mathrm{~kg}$.

Nous injectons d'emblée $400 \mathrm{mg}$ du tranquillisant. L'effet total de détente se produit en 30 minutes.

Les animaux sont conduits avec la plus grande facilité dans une petite embarcation par une seule personne. A destination, ils sont débarqués sans aucune ciffficulté.

Un homme seul réussit à scier les cornes des deux buffles sans qu'il faille recourir à la moindre contiention.

L'acépromazine agit pendant 8 heures environ.

Commentaires. - La grosse difficulté consiste à ir'ecter le produlit sur des animaux sauvages. D as-ès une documentation que nous avons reçue ves U.S.A. University of Georgia), il est possible da áministrer les tranquillisants à l'aide d'une serirgue projectile automatique, qui peut être ancée par un « long range projector » à une distance de 30 mètres, l'opération demandant $b$ secorves. Lutilisation d'une telle méthode paraît particulièrement digne d'intérêt. 


\section{2. - CHEZ LES ZEBUS}

\section{A) Red-Sindhi}

Les essais ont porté sur deux taureaux, trois vaches ef deux génisses. Nous sommes intervenu à la demande du directeur des Services agricoles sur des animaux qui étaient devenus particulièreme.t farouches à la suite d'un long voyage.

Taureaux. - $\|_{s}$ pèsent environ 600 et $700 \mathrm{~kg}$. Nous administrons $250 \mathrm{mg}$ en intra-musculaire. Vingt minutes plus tard, ils sont facilement débarqués du bateau ef chargés sur un camion.

Dix minutes après être arrivés à destination, ils regagnent avec une grande difficulté l'abri qui leur est réservé; on a l'impression qu'ils dorment debout. Ils se couchent immédiatement et nous les retrouvons en décubitus complet lors de la visite effectuée le lendemain matin, soit 12 houres après. Sous l'effet de l'aiguillon électrique, ils font des efforts pour se relever, mais sans succès.

Le même jour, vers midi, its sont toujours couchés mais se nourrissent.

Au bout de 48 heures, ils sont encore dans la même situation.

Trois jours àprès l'intervention, dans la matınée, nous les trouvons debout, occupés à brouter, et dans un état normal.

Vaches. Nous sommes en présence de trois sujets pesant $400,450,500 \mathrm{~kg}$ auxquels nous injectons $200 \mathrm{mg}$.

Vingt minutes après, les vaches sont complètement « détendues». Transportées à leur lieu de destination au bout de 30 minutes, elles parcourent avec peine les $50 \mathrm{~m}$ qui séparent le lieu de débarquement de leur abri ; elles dorment debout.

Le lendemain matin, elles sont toutes trois couchées et nous montrent les troubles nerveux suivants:

10 Spasme tonique des muscles anconés:

$2^{\circ}$ Spasme clonique des muscles des lèvres et des paupières ;

$3^{0}$ Balancements rythmiques de la tête.

Soumises à l'excitation de l'aiguillon électriaue, elles se relèvent immédiatement et nous notons une exacerbation du syndrome spastique.
Ces symptômes persistent pendant deux jours, durant lesquels les animaux mangent peu.

Le troisième jour, ils sont redevenus normaux.

Génisses. - Elles pèsent chacune $200 \mathrm{~kg}$ ef reçoivent $100 \mathrm{mg}$ de tranquillisant. Aucun phénomène de relaxation ne se produit. Les deux animaux sont difficilement maîtrisés par les gardiens.

\section{B. Gyr}

Trente animaux assez farouches devaient être transportés sur de petites embarcations pour être transférés par la rivière à $30 \mathrm{~km}$ plus loin, clans des conditions difficiles; il nous était nécessaire de recourir à l'acépromazine.

Il serait fastidieux de décrire le comportement de chaque animal; nous nous bornerons à relever les remarques suivantes:

Comportement des animaux. - L'administration d'une même dose sur des animaux de même poids n'est pas toujours suivie d'un effet identique. Ainsi, des animaux farouchement méchants se montrent plus calmes après l'intervention que d'autres naturellement moins farouches.

Réactions. - Sur des animaux n'ayant reçu que $100 \mathrm{mg}$ de maléate acide d'acépromazine. nous avons constaté des troubles de vertigo, persistant pendant environ 4 heures.

\section{Réflexes.}

a) Réflexes cutanés. - Ils sont testés par le toucher de l'anus. Sur certains animaux bien tranquillisés, les contractions du sphincter sont nulles; sur d'autres, elles restent normales.

b) Réflexe pupillaire. - N reste constant sur tous $k \in S$ animaux.

Toxicité. - Outre les troubles nerveux mentionnés à propos des Red-Sindhi, nous mentionnerons un cas de mortalité survenu dáns les circonstances suivantes: Une vache Gyr de $300 \mathrm{~kg}$, très méchante, reçoit $100 \mathrm{mg}$ de tranquillisant; au bout d'une heure, aucun effet n'est apparent: après une attente supplémentaire de 30 minutes, nous injectons une nouvelle dose de $100 \mathrm{mg}$; 20 minutes plus, tard, la vache se couche et nous remarquons alors une dépression respiratoire accompagnée d'un collapsus circulatoire; le lendemain matin, elle a cessé de vivre. 
Durée de l'effet du tranquillisant. - Le produit agit totalement pendant 6 à 8 heures, pour disparaitre complètement 14 heures après flintervention.

\section{CONCLUSION}

II эpparaît àinsi que l'emploi du maléate acide d'acépromazine est particulièrement précieux lorsque l'on est obligé de maîtriser ou de manipuler aes animaux aussi dangereux que des zébus ou des buffles.

Le táit de ne pas être obligé de recourir à l'iniection intraveineuse facilite l'intervention. L'effat tranquillisant est précoce et durable. La dose à employer chez le buffle doit être deux fois plus élevée que chez les bovins. Cependant it ccivient d'observer la plus grande prudence en ce qui concerne la posologie de ce produit.

Services vétérinaires de la Guyane française.

\section{SUMMARY}

\section{Use of maleate Salt of promazine as a bovine tranquiliser.}

Observations show the value of the maleate salt of promazine when handling indocile or dangerous animals. The injection is given intramuscularly. The tranquilizing effect is quick and lasting, but sometimes inconstant. Great care must be given to the posology of this product. In 40 cnimals treated ( 3 buffaloes and 37 zebu cattle) one of the latter died following a dose $200 \mathrm{mg}$. for a liveweight of $300 \mathrm{kgs}$. In some animals the product has no effect. The author used a double dose for buffaloes $(400 \mathrm{mg}$.$) as compared to zebu cattle.$

\section{RESUMEN}

\section{Utilización del maleato ácido de acepromazina como tranquilizador en los grandes rumiantes.}

Las observaciones aportadas muestran el interés que presanta el maleato ácido de aceprcmazina zuando es necesario dominar o manipular animales peligrosos o indóciles. Le inyección se efectía por vía intramuscular; el efecto tranquilizador es precoz y duradero, pero a veces inconstante; conviene usar la mayor prudencia en lo concerniente a la posología de este producto. De 40 animales tratados ( 3 búfalos y 37 cebús), una vaca sucumbió (dosis de 200 mgr. para un peso de $300 \mathrm{~kg}$ ). y en algunos animales el producto se inanifestó sin efecto. El autor ha utilizado una dosis doble para los búfalos $(400 \mathrm{mgr})$, en relación a la de los cebús. 